\title{
Lateral Mass Screw Fixation in the Cervical Spine: Introducing a New Technique
}

\author{
Sreeramalingam Rathinavelu, Ariful Islam, Pankaj Shivhare, Sandip Chatterjee \\ Department of Neurosurgery, Park Clinic, Kolkata, India
}

Study Design: This was designed as a randomized double blind study to compare the classical Magerl technique of insertion of lateral mass screws with the authors' technique. The observations regarding length, outcome, and radiology was done by a group blinded to the technique used.

Purpose: The present study was designed with the objective of identifying the optimal technique for introducing the lateral mass screws that uses the maximum possible dimension of the lateral mass.

Overview of Literature: Lateral mass screw fixation is a common surgery that is performed in the cervical spine. Various modifications for the procedure have been described, such as changes in the entry point, angulation of the screws, and modifications in the exit point. These do not allow the insertion of longer screws that can give more purchase on the bone.

Methods: From January 1, 2009 to December 31, 2018, 176 patients who were scheduled to undergo lateral mass screw fixation were enrolled. They were randomized into two groups; we inserted lateral mass screws using our new technique for one group and by using the classical Magerl technique for the other group. Intraoperative measurements were used to assess the bone-screw interface length. Postoperative radiography and postoperative computed tomography were performed to assess the trajectory of the screws.

Results: Total 88 patients were included in the study group, including 68 men. The control group included 65 men. The most common indication for surgery was cervical spondylotic myelopathy. The average bi-cortical length that was measured intraoperatively was $19.9 \mathrm{~mm}$ in the study group and $16.3 \mathrm{~mm}$ in the control group. This was significantly different from the average lengths of screws in the control group.

Conclusions: The trajectory that involves an entry point as close as possible to the posterior inferior medial angle of the lateral mass cuboid and traverses a distance of about $20 \mathrm{~mm}$ to obtain a bi-cortical purchase in the diagonally opposite angle may provide a much better and firmer bony purchase in the lateral mass than conventional points of entry and trajectories.

Keywords: Magerl technique; Myelopathy; Entry point; Trajectory; Screw; Spinal cord disease

\section{Introduction}

Currently, lateral mass screw fixation in the cervical spine is one of the most common surgeries performed in the cervical spine. Since 1972, many modifications of the procedure have been described. The variations include a change in the entry point, variation in the angulation of the screws, and differences in the exit point. The most

Received Apr 2, 2020; Revised Jul 9, 2020; Accepted Jul 18, 2020

Corresponding author: Sandip Chatterjee

Department of Neurosurgery, Park Clinic, 4 Gorky terrace, Kolkata 700017, India

Tel: +91-3322800758, Fax: +91-3322801807, E-mail: parkclkinic@gmail.com 
popular among these techniques are those described by Roy-Camille et al. [1] and Jeanneret et al. [2]. Various other authors, including An et al. [3] and Kim et al. [4] have suggested modifications of these techniques. One common characteristic of all these techniques is the objective of ensuring safety of the neurovascular structures lying anterior and anterolateral to the lateral mass. These improvements have made lateral mass screw fixation in the cervical spine a safer technique; however, they do not allow the insertion of longer screws that can give more purchase on the bone. Furthermore, intraoperatively, we face lateral cutout of the screws resulting in fracture of the lateral mass.

The objective of this study was to identify an alternative technique for introducing the lateral mass screws that use the maximum possible dimension of the lateral mass and ensure a certain degree of safety as provided by other techniques.

\section{Materials and Methods}

\section{Study population}

From January 1, 2009 to December 31, 2017, 176 patients who were scheduled to undergo lateral mass screw fixation were enrolled. They were randomized into two equal groups; for the study group, lateral mass screws were inserted with our new technique and for the control group, the screws were inserted using the classical Magerl technique. The study was approved by the Institutional Review Board of Park Clinic (IRB approval no., CA23/202). Informed consent was obtained from all patients enrolled in the study.

\section{Surgical technique}

The patients were made to lie in the prone position with their head stabilized over the horseshoe frame. An image intensifier was used to confirm the alignment. Midline skin incision was used, and sub periosteal dissection was performed till the outer edge of the lateral mass. The four borders of the lateral mass were identified. Upper and lower borders were defined by the superior and inferior facet joints. The medial border was defined by the junction of the lamina; the lateral mass and lateral border were defined by the outer edge of the lateral mass.

\section{Entry point}

The chosen entry point was the point of intersection of lines drawn $2 \mathrm{~mm}$ lateral to the medial border of the lateral mass and $2 \mathrm{~mm}$ above the inferior facet joint. Fig. 1 illustrates the insertion point in a bone model.

\section{Trajectory}

Minimal shaving of the spinous process of the inferior vertebrae was required for proper instrument placement. We preferred to use a drill to create the entry point. Initially, until the proximal cortex was pierced, the drill was

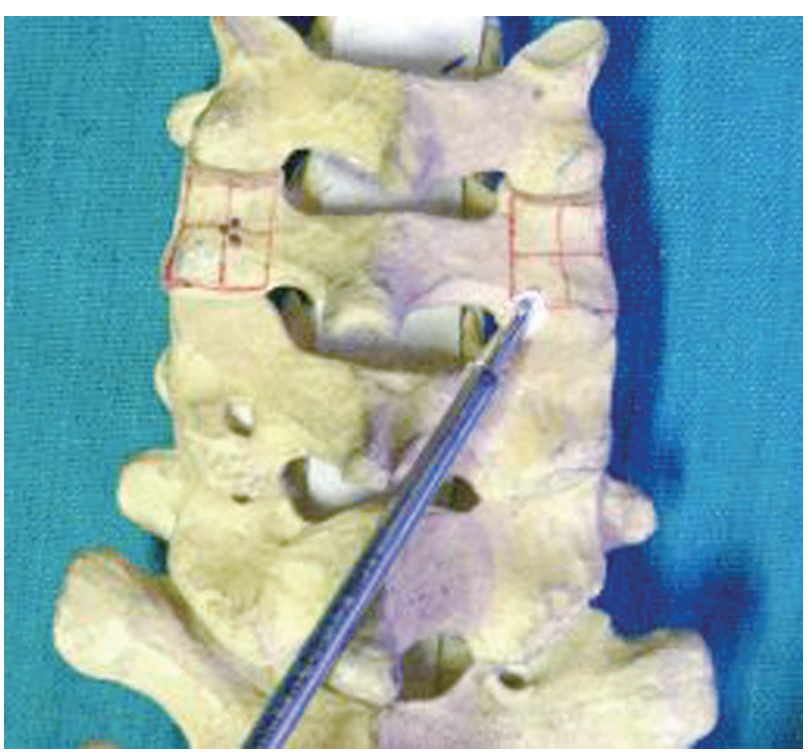

Fig. 1. Showing the insertion point in a bone model.

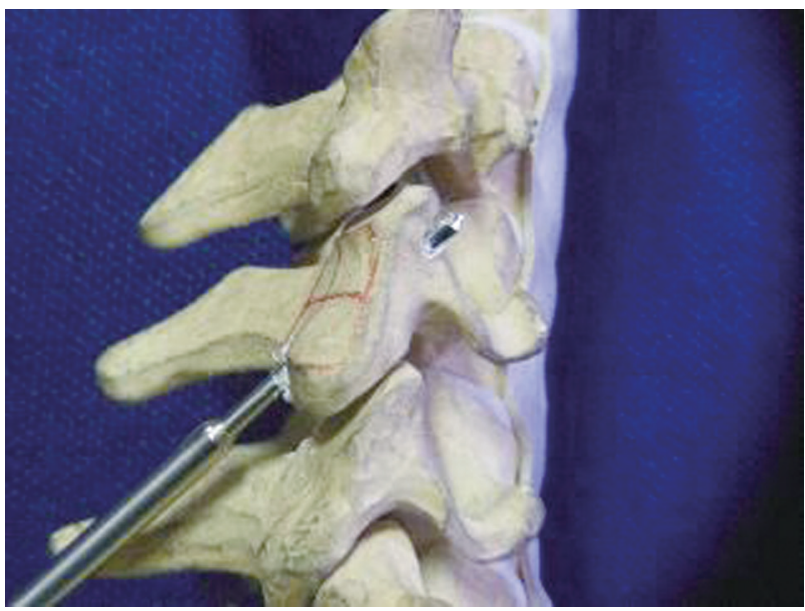

Fig. 2. Showing the entry point, trajectory, and exit point used in our technique. 
angled perpendicular to the surface. Once the cortex was crossed, an awl was used and was aimed at the upper outer quadrant of the lateral mass, with care being taken to never angulate less than the angle of the lamina of the same bone laterally. This helped in avoiding the path of the vertebral artery. The shaft of the awl should cross the midline at the level of the spinous process. This provided an anteroposterior direction to reach the diagonally opposite corner of the lateral mass. It is necessary to angulate more than the angle of the upper facet joint to avoid facet joint breach. The exit point from the lateral mass was the same as that in the Magerl technique. This lowered the chances of nerve injury. Fig. 2 shows the entry point, trajectory, and exit point used in the study group. The outer cortex was pierced with an awl or using diamond drill. The path of the trajectory was felt with a ball tip probe. Probes were inserted into all the lateral masses, and lateral alignment was confirmed using an image intensifier.

\section{Screw length}

The screw length was measured using the same ball tip probe. The length of the probe when it just hooks the outer pierced cortex was measured, and screws with the same length were inserted. We used a $2.5-\mathrm{mm}$ tap to prepare the trajectory and drilled the periphery of the proximal entry area for the screw heads to sit properly. We used 3.5$\mathrm{mm}$ screws for all the patients. The poly-axial screws that are used should have around $60^{\circ}$ angulation if possible. Screws that do not permit sufficient angulations should

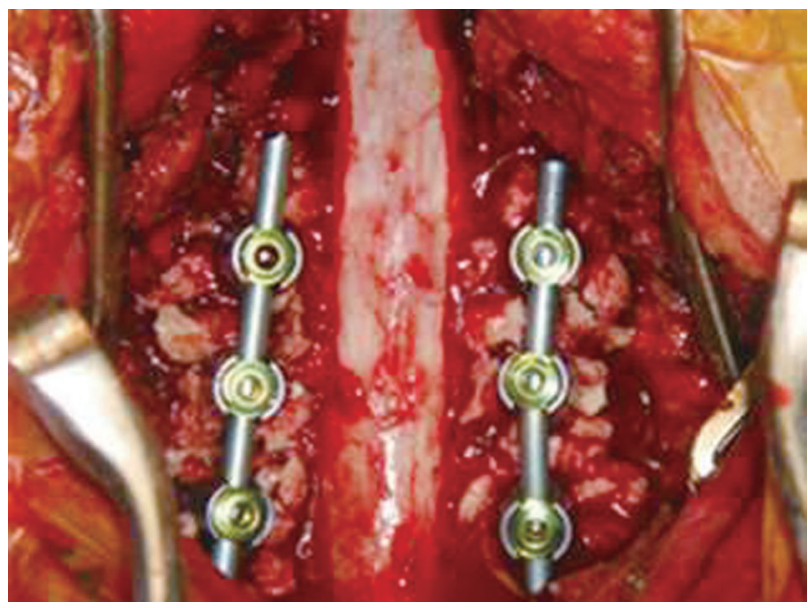

Fig. 3. Operative photograph of the lateral mass screw and rod fixation. be avoided because they tend to lift the lower border during rod reduction. Bi-cortical purchase was ensured in all patients. The final screw placement was always confirmed using an image intensifier.

\section{Rod placement}

Rods were contoured to the required angulation and fixed to the screws using the inner screws. Some force is acceptable while using our technique. Fig. 3 shows the operative photograph of the lateral mass screw and rod fixation and Fig. 4 depicts anteroposterior and lateral view of the peroperative image of lateral mass screw in the C-arm.

\section{Clinical and radiological evaluation}

Neurological assessment of the patient was performed at the time of admission and after the surgery. Only patients who presented with cervical myelopathy were included, and those with radiculopathy were specifically excluded. The intraoperative screw length, any fracture of the lateral mass, fracture of the proximal cortex at entry point, screw pullout during rod fixation, loosening of the construct, vertebral artery injury, and other screw-related events were noted. Symptoms related to nerve root irritation or injury was checked during the postoperative period.

All the patients underwent cervical spine anteroposterior and lateral radiography and magnetic resonance imaging as part of the preoperative workup. Postoperatively, anteroposterior and lateral radiography and computed tomography (CT) scan of the cervical spine were performed as part of the protocol to assess the trajectory of the screws. Fig. 5 demonstrates the postoperative images of radiography and CT scan of in-situ lateral mass screw. Violation of the facet joint and screw protrusion in the
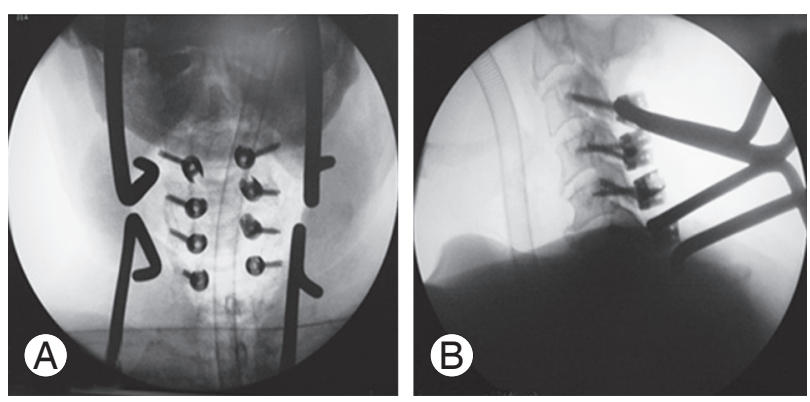

Fig. 4. (A, B) Anteroposterior and lateral view of peroperative image of lateral mass screw in $\mathrm{C}$-arm. 

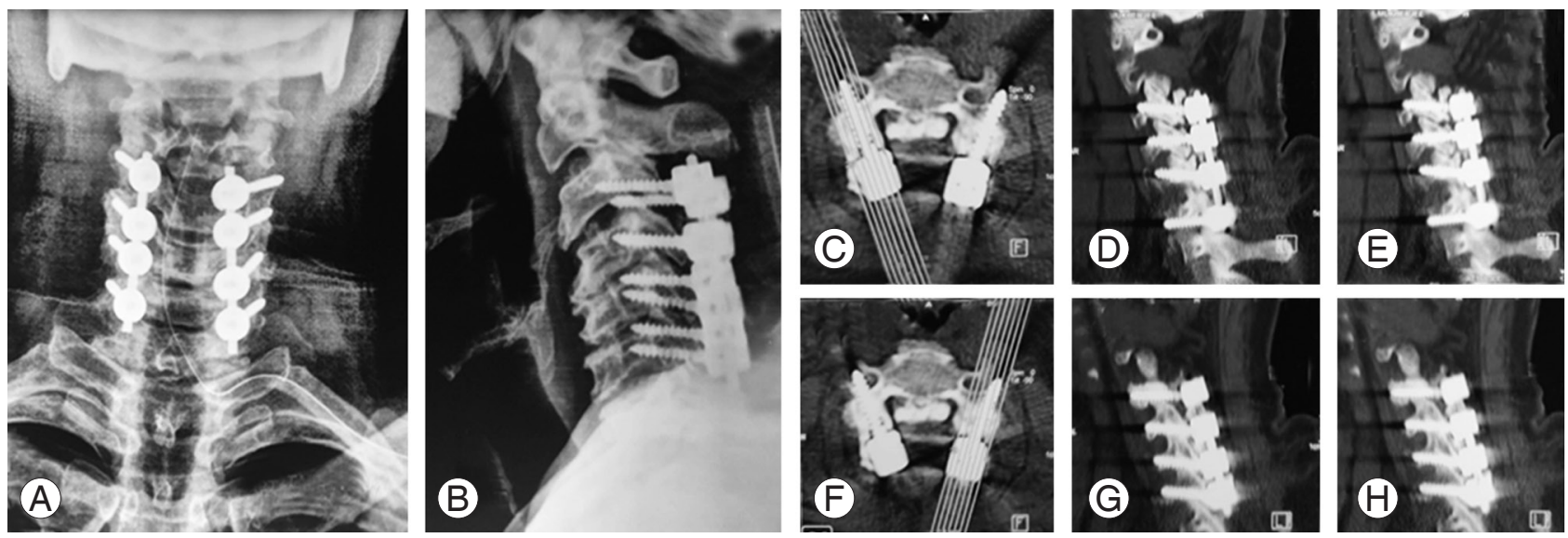

Fig. 5. Postoperative image of $\mathbf{X}$-ray (A, B) and computed tomography scan (C-H) of in-situ lateral mass screw.

transverse foramen were specifically noted. Follow-up was scheduled at 3, 6, and 12 months. Fusion was considered clinically successful if there was no movement on the flexion-extension lateral views. During follow-up visits, symptoms related to implant failure, such as implant loosening, screw breakage, screw pull out, and construct failure, were noted.

\section{Results}

Total 88 patients were enrolled in the study group, including 68 men and 20 women, with an average age of 59 years. Of the 88 subjects in the control group, 65 were men. Total 664 screws were inserted. The most common indication for surgery was cervical spondylotic myelopathy. Other etiologies were ossification of the posterior longitudinal ligament in 28 patients, trauma in 16, and tuberculosis in 12 . The average length of the screw in the study group was $19.9 \mathrm{~mm}$. Details of the screw lengths are shown in Table 1. This was significantly different from the average lengths of the screws in controls. The screw length was significantly different between the two groups.

In the study group, no screws violated the transverse foramen, and no intraoperative vertebral artery injury was observed in any patient. None of the patients developed symptoms/signs of nerve root irritation during the postoperative period. There was no case of lateral mass fracture. Proximal cortex split at the entry point was noted in two screws. Screw pullout occurred during rod reduction in one patient. This patient was operated for Pott's paraparesis with posterior element involvement. The involved $\mathrm{C} 4$ level was skipped; C3, C5, and C6 were fixed on the left
Table 1. The mean length of the screw in the study group and control group

\begin{tabular}{lccc} 
& $\begin{array}{c}\text { Our technique: study } \\
\text { group (mm) }\end{array}$ & $\begin{array}{c}\text { Magerl technique: control } \\
\text { group (mm) }\end{array}$ & $p$-value \\
C3 & $19.7 \pm 2.8$ & $16.0 \pm 1.4$ & $<0.0001$ \\
C4 & $20.0 \pm 1.7$ & $16.2 \pm 1.6$ & $<0.0001$ \\
C5 & $20.1 \pm 1.7$ & $16.8 \pm 1.4$ & $<0.0001$ \\
C6 & $19.9 \pm 1.5$ & $17.7 \pm 0.8$ & $<0.0001$ \\
\hline
\end{tabular}

Values are presented as mean \pm standard deviation.

side, and C3, C4, C5, and C6 were fixed on the opposite side. A crosslink was used, and as the construct was holding well, we decided to wait and watch. His postoperative and follow-up course was uneventful, and he recovered well. One patient had temporary C5 root palsy, and the condition improved at 3 months. This could be due to traction on the root by posterior movement of the cord, not due to screw placement. Facet joint violation was observed in six screws. No demonstrable instability was observed on any of the follow-up flexion-extension lateral views. No case of screw breakage, loosening, or late implant failure was noted. In contrast to that in the control group, the incidence of proximal cortical split and screw pullout was definitely higher, at 8 and 4, respectively. One patient had temporary C5 nerve palsy and 17 had violation of facet joints.

In the control group, two screws violated the transverse foramen; however, there were no vertebral artery injuries. Two patients developed signs of nerve irritation, and there were five lateral mass fractures. Proximal cortical split was observed in 11 screws. 


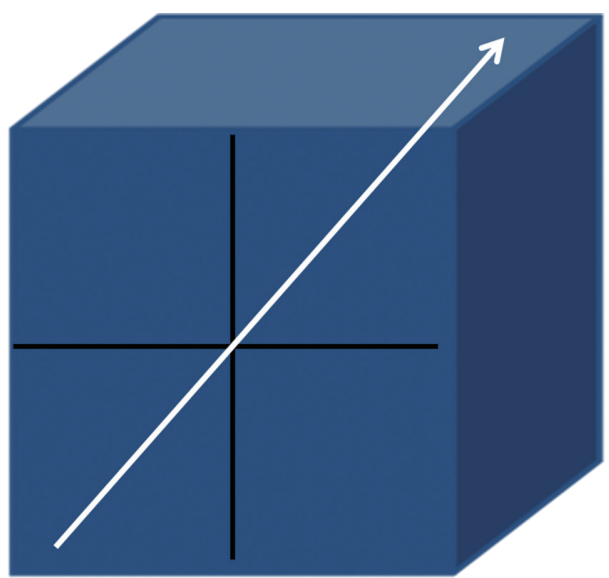

Fig. 6. Showing the geometrical representation of the trajectory used.

\section{Discussion}

Lateral mass screw fixation is accepted as a relatively safe and reliable technique for posterior stabilization of the sub axial cervical spine. Roy-Camillie et al. [1] introduced the technique in 1972. Thereafter, several modifications have been suggested by many authors. All these modifications aimed to improve the safety margin of this technique. Their main considerations were vertebral artery injury, nerve root injury, facet joint violation, and fusion. Cervical pedicle screws score ahead of them when the pullout strength is taken into consideration. But as far as safety in concerned lateral mass screws provide a stronger fixation which is also safe.

Fig. 6 shows the geometry of the lateral mass would suggest, the longest screw possible will be the one that lies diagonally from postero-medial-inferior corner to the antero-lateral-superior corner. This, if possible, will give the maximum possible bone purchase; further, it is starting well medial to the lateral border; therefore, it should decrease the lateral cortical fracture. With this principle in mind, we have planned out the technique. The entry point was chosen in the postero-medial-inferior quadrant. While choosing the entry point, care was taken to avoid injury to the lower facet joint by avoiding $2 \mathrm{~mm}$ from the lower border of the lateral mass. The medial point entry was about $2 \mathrm{~mm}$ from the lamina-lateral mass junction because the lamina always ends at an angulation to the lateral mass, and any instrument used for drilling laterally from that point will lean on the lamina and cannot be used from the exact junction. There are no major safety concerns in the posterior aspect; therefore, the entry point was chosen $2 \mathrm{~mm}$ lateral to the medial border and $2 \mathrm{~mm}$ from the lower border.

Quadrant anatomy of the lateral mass was discussed in detail by Pait et al [5]. They have measured the medial facet line, the vertebral artery line. They found great variance in measurements not only between the different spines, but also between different levels in the same spine. The only consistent result in their study was that no neurovascular structures were present under the superolateral quadrant. They considered this quadrant as the "safe quadrant" for the placement of posterior screws and plates. In a cadaveric study, 9.3-12.2 $\mathrm{mm}$ was the average distance between the posterior midpoint of the lateral mass and the vertebral foramen from C3 to C6 [6]. In fact, as far as the entry point is concerned, there are only few safety factors; however, the exit point is crucial owing to the presence of neurovascular structures. This forms the basis for the exit point suggested by us. Exiting anywhere in the superolateral quadrant anteriorly or laterally is safe.

One of the concerns raised by Xu et al. [7] on the exit point of Magerl technique was that regarding nerve root violation. One nerve root injury was observed in each cervical spine segment using the Roy-Camille method (8.3\%) and the Magerl method (5.6\%) in another cadaveric study by Baek et al. [8]. However, Jeanneret et al. [2] has reported only two cases of nerve root-related problems in their total 51 patients. Further, these two cases were not related to fixation. The incidence of nerve root injury was reported at $0.6 \%$ by Heller et al. [9] who concluded that although cadaveric studies had predicted more complications, the actual risk in the clinical setting is lower. Wellman et al. [10] investigated 281 screws without any nerve root injury. In our study also, there was no root injury related to fixation.

Vertebral artery injury remains a major concern; however, the reported prevalence rate is negligible $[9,10]$. Sureisen et al. [11] published their study on the radiological evaluation of lateral mass screw angulation in an Asian population to avoid vertebral artery canal penetration. Their minimum mean value was $6.2^{\circ}$ at $\mathrm{C} 4$ with the use of the Roy-Camille entry point, and the maximum mean value was $14.3^{\circ}$ at $\mathrm{C} 6$ with the use of the Magerl entry point. However, the angulations suggested for the same entry points are much more, at $10^{\circ}$ and $25^{\circ}$, respectively $[1,2]$. In fact, inserting screws with exact angulations intraoperatively is not easy. Most surgeons have their own landmarks for angulations. We suggest aiming at exiting 
in the superolateral quadrant, which is not difficult. We also recommend caution while using our method so as to never angulate less than the angle subtended by the same side lamina to the horizontal. This is important for avoiding the vertebral artery because the transverse foramen is almost always present inside a straight line drawn from the outer aspect of the same side lamina of the same vertebra.

Safe screw lengths for Roy-Camille and Magerl techniques have been described by Ebraheim et al. [12] in their cadaveric study on 14 cervical spines. They suggested a screw length of $14-15 \mathrm{~mm}$ in the Roy-Camille technique and $15-16 \mathrm{~mm}$ in the Magerl technique. This difference in screw length was attributed to the oblique angle used in the Magerl technique. They have suggested still shorter screws in C7. In their study on a Korean population, Cho and Kim [13] recommend a length of 13.5 $\mathrm{mm}$ from $\mathrm{C} 3$ to $\mathrm{C} 6$ and $13 \mathrm{~mm}$ at C7 for the Roy-Camille technique and $14 \mathrm{~mm}$ from $\mathrm{C} 3$ to $\mathrm{C} 6$ and $13.6 \mathrm{~mm}$ at $\mathrm{C} 7$ for the Magerl technique. Yoon et al. [14] did a radiological study on the Roy-Camille and Magerl techniques. In the study, the Magerl method showed a mean screw depth of $11.9 \pm 1.5 \mathrm{~mm}$, while the Roy-Camille method showed a screw depth of $10.5 \pm 1.4 \mathrm{~mm}$. When using a method of Baek et al. [8], the ideal length for bi-cortical purchase ranged from 13.7 to $13.9 \mathrm{~mm}$, and these authors have used 14-mm screws uniformly. Our average screw length was $20 \mathrm{~mm}$, about $4-6 \mathrm{~mm}$ more than that recommended for other techniques. Thus, we can use a screw that is $20 \%-30 \%$ longer and has 20\%-30\% more bone purchase than that in the previous techniques. The maximum and minimum screw size was $24 \mathrm{~mm}$ and $18 \mathrm{~mm}$, respectively, in our series.

In their study on the biomechanical pullout strength of lateral mass screws, Heller et al. [15] have shown bi-cortical purchase to be the single most important factor that significantly improves the pullout strength of lateral mass screws when conventional methods of insertion are used. As per them, the strength of bi-cortical purchase of screws is $30 \%$ more than that of uni-cortical purchase. The greatest pullout resistance was at C4 level. Choueka et al. [16] studied the influence of insertion technique and position on flexion failure of posterior cervical lateral mass screws and showed a significant correlation between screw path length and load to failure at superior screw hole positions.

Further, they found that in relation to implant failure, there is significant correlation between vertebral body bone mineral densities and screw positions ${ }^{4}$. Both these studies were cadaveric studies. Seybold et al. [17] has reported no significant difference in the pullout strength between uni-cortical and bi-cortical screws. Although there is evidence in support of both, we believe that a bi-cortical purchase is better than a uni-cortical purchase. We did not perform a biomechanical study with our technique; however, we believe that an increase of $20 \%-30 \%$ bone purchase with $100 \%$ bi-cortical purchase in every screw will improve the pullout strength of the overall construct.

Lateral mass fracture on screw insertion was $6 \%$ with Roy-Camille screws and with 7\% with the Magerl screws in the cadaveric study by Choueka et al. [16]. In 2007, Kim et al. [18] reported two lateral mass fractures wherein they changed the trajectory and used 4-mm screws for rescue. We did not observe any case of lateral mass fracture in our study group. We attribute this to our more medial entry point that allows a strong lateral part to support during drilling and screw placement.

At C7, we use pedicle screws owing to the small lateral mass and greater risk of injuring the $\mathrm{C} 8$ root. Moreover, with an empty vertebral artery foramen at $\mathrm{C} 7$ and a better pedicle hold, definitely pedicles are stronger and safer at C7. We did not use our method at C7 level. All the patients were operated at the same center by a senior surgeon. A multi-centric study may reveal more complications or technical challenges that were not encountered during our study.

\section{Conclusions}

A trajectory that involves an entry point in the posterior inferior medial angle of the lateral mass cuboid and traverses a distance of about $20 \mathrm{~mm}$ to obtain a bi-cortical purchase in the diagonally opposite angle can provide a much better and firmer bony purchase in the lateral mass than conventional points of entry and conventional trajectories that achieve a much shorter screw length. Moreover, the confirmed safety of this technique makes us recommend it to every surgeon who wants to insert lateral mass screws. The screw lengths using this technique were about $30 \%$ longer. We would like to recommend this technique of insertion of lateral mass screws in the cervical spine as being biomechanically superior and safer than the conventional techniques. 


\section{Conflict of Interest}

No potential conflict of interest relevant to this article was reported.

\section{Author Contributions}

Collection of the data, and consenting for study: Sreeramalingam Rathinavelu, Ariful Islam; overall data collection and analysis of the data: Pankaj Shivhare; design and overall execution of the study: Sandip Chatterjee.

\section{References}

1. Roy-Camille R, Saillant G, Laville C, Benazet JP. Treatment of lower cervical spinal injuries: C3 to C7. Spine (Phila Pa 1976) 1992;17(10 Suppl):S442-6.

2. Jeanneret B, Magerl F, Ward EH, Ward JC. Posterior stabilization of the cervical spine with hook plates. Spine (Phila Pa 1976) 1991;16(3 Suppl):S56-63.

3. An HS, Gordin R, Renner K. Anatomic considerations for plate-screw fixation of the cervical spine. Spine (Phila Pa 1976) 1991;16(10 Suppl):S548-51.

4. Kim SH, Seo WD, Kim KH, Yeo HT, Choi GH, Kim DH. Clinical outcome of modified cervical lateral mass screw fixation technique. J Korean Neurosurg Soc 2012;52:114-9.

5. Pait TG, McAllister PV, Kaufman HH. Quadrant anatomy of the articular pillars (lateral cervical mass) of the cervical spine. J Neurosurg 1995;82:1011-4.

6. Ebraheim NA, Xu R, Yeasting RA. The location of the vertebral artery foramen and its relation to posterior lateral mass screw fixation. Spine (Phila Pa 1976) 1996;21:1291-5.

7. Xu R, Haman SP, Ebraheim NA, Yeasting RA. The anatomic relation of lateral mass screws to the spinal nerves: a comparison of the Magerl, Anderson, and An techniques. Spine (Phila Pa 1976) 1999;24:205761.

8. Baek JW, Park DM, Kim DH. Comparative analysis of three different cervical lateral mass screw fixa- tion techniques by complications and bicortical purchase: cadaveric study. J Korean Neurosurg Soc 2010;48:193-8.

9. Heller JG, Silcox DH 3rd, Sutterlin CE 3rd. Complications of posterior cervical plating. Spine (Phila $\mathrm{Pa}$ 1976) 1995;20:2442-8.

10. Wellman BJ, Follett KA, Traynelis VC. Complications of posterior articular mass plate fixation of the subaxial cervical spine in 43 consecutive patients. Spine (Phila Pa 1976) 1998;23:193-200.

11. Sureisen M, Saw LB, Wei Chan CY, Singh DA, Kwan MK. Radiological assessment of cervical lateral mass screw angulations in Asian patients. Indian J Orthop 2011;45:504-7.

12. Ebraheim NA, Klausner T, Xu R, Yeasting RA. Safe lateral-mass screw lengths in the Roy-Camille and Magerl techniques: an anatomic study. Spine (Phila Pa 1976) 1998;23:1739-42.

13. Cho JI, Kim DH. Comparative analysis of cervical lateral mass screw insertion among three techniques in the Korean population by quantitative measurements with reformatted 2D CT scan images: clinical research. J Korean Neurosurg Soc 2008;44:124-30.

14. Yoon SH, Park HC, Park HS, et al. Radiological considerations of posterior cervical lateral mass fixation using plate and screw. Yonsei Med J 2004;45:406-12.

15. Heller JG, Estes BT, Zaouali M, Diop A. Biomechanical study of screws in the lateral masses: variables affecting pull-out resistance. J Bone Joint Surg Am 1996;78:1315-21.

16. Choueka J, Spivak JM, Kummer FJ, Steger T. Flexion failure of posterior cervical lateral mass screws: influence of insertion technique and position. Spine (Phila Pa 1976) 1996;21:462-8.

17. Seybold EA, Baker JA, Criscitiello AA, Ordway NR, Park CK, Connolly PJ. Characteristics of unicortical and bicortical lateral mass screws in the cervical spine. Spine (Phila Pa 1976) 1999;24:2397-403.

18. Kim SH, Shin DA, Yi S, Yoon DH, Kim KN, Shin HC. Early results from posterior cervical fusion with a screw-rod system. Yonsei Med J 2007;48:440-8. 